\title{
Intracellular Transport of HIV-1 Matrix Protein Associated with Viral RNA
}

\author{
Anatoliy I. Gozhenko ${ }^{1}$, Valentina A. Divocha ${ }^{1}$, Galina K. Vorkunova², Alissa G. Bukrinskaya ${ }^{2}$, \\ Sergey I. Lupandin ${ }^{2}$ \\ ${ }^{1}$ School State Ukrainian Research Institute of the Medicine on Transport, Odessa, Ukraine; ${ }^{2}$ Ivanovsky Institute of Virology, Mos- \\ cow, Russia. \\ Email: bukrinskaya@yandex.ru
}

Received November $4^{\text {th }}$, 2012; revised December $23^{\text {rd }}, 2012$; accepted January $4^{\text {th }}, 2013$

\begin{abstract}
HIV-1 matrix protein (MA) is a multifunctional structural protein localized on N terminus of Gag precursor p55. MA participates in HIV-1 assembly as membranotropic part of Gag precursor as well as an individual protein spliced from Gag early in infection. MA is found in the nuclei of infected cells and in plasma membrane, the site of virus assembly, in association with viral genome RNA. MA mutated variant M4 which contains two changed amino acids in N-terminal regions is also associated with viral RNA, but it is localized in the nuclear and cytoskeleton fractions but not in the plasma membrane suggesting that the mutant is deprived of membranotropic signal and "sticks" in the nuclei an $d$ cytoskeleton, its previous location sites. These data allow suggesting that MA involved into transmission of viral RNA is transported to plasma membrane by cytoskeleton.
\end{abstract}

Keywords: HIV-1 Matrix Protein; Gag Precursor P55; Cytoskeleton; Viral RNA; Transport of Viral Complex; Plasma Membranes; Cell Fractionatiomn

\section{Introduction}

Matrix protein p17 is the smaller protein of HIV-1 but it plays a lot of functions in viral life cycle. MA possessis three transport signals and is involved in interaction with Gag protein-the main protein in viral assembly. Its presence in the nuclei could be connected with the formation of the complex with the viral RNA as shown earlier [1]. We studied this possibility by comparing the intracellular localization of standart MA and mutant MA in complex with viral RNA.

HIV-1 assembly process is driven and coordinated by Gag precursor p55 which is generally sufficient for the assembly of virus-like particles. An essential step of HIV-1 assembly is the interaction of viral genome RNA (gRNA) with Gag polyprotein, however, it is still unknown when and where viral genome RNA is transported from the nuclei to plasma membrane and is associated with Gag precursor.

The membranotropic part of Gag on N-terminal is matrix protein p17 (MA). It possesses three transport signals -membranotropic, nucleophy lic and the signal of nuclear export [2] and is regarded as a shuttling protein in infected cells. MA could be spliced from Gag precursor early in infection [3] but the authors believed that early spliced viral proteins did not function in infected cells. We suggested that early matrix protein could be involved in viral life cycle [4] and particulary in virus assembly, and tried to determine MA intracellular localization, it association with viral RNA and the way of thear intracellular transport.

Figure 1(a) shows the result of pulse-chase experiment using HIV-1 infected MT4 cells. 14C leucine was added 20 hours after infection for 20 minutes (pulse) and the label was chased for 1, 2 and 3 hours. Cells were fractionated as described [1] to get the fractions of the cytosol, membranes and nuclei. It is seen that during chase the amount of Gag precursor p55 in cytosol fraction is diminished and after chase the protein is found in membrane fraction (the place of virus assembly). Meanwhile, the amount of matrix protein in cytosol during the chase is increasing suggesting that matrix protein is spliced from Gag precursor soon after its synthesis. The large amount of $120 \mathrm{~S}$ glycoproteins show that they are early synthesized and quickly transported to the membranes.

It has been shown that during infection matrix protein penetrates into the nuclei [5] and viral RNA is accumulated in the nuclei following pulse-chase method and the longer (for 3h) labeling of the infected cells with 14C leucine (Figure 1(b)). MA has been found in the nuclear and membrane fractions of MT4 cells in association with viral RNA including genome RNA using slot and blot 


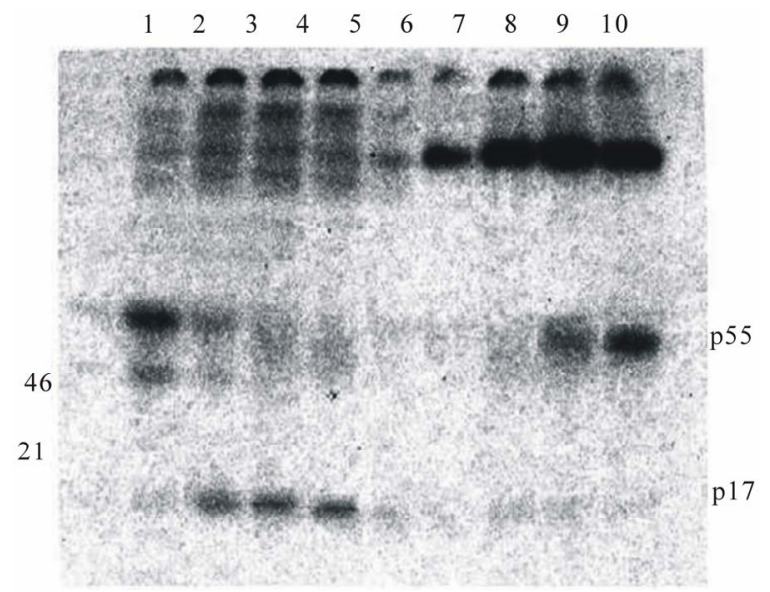

(a)

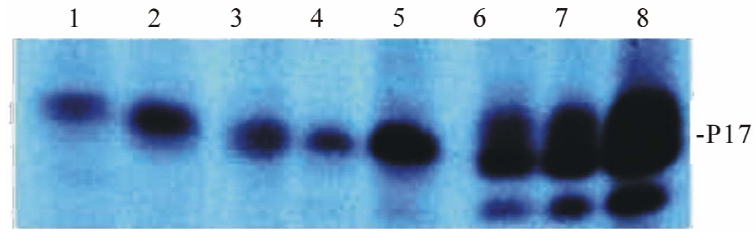

(b)

Figure 1. Localization of viral proteins in cellular fractions. Pulse-chase experiment. (a) MT4 cells were infected with HIV-1 virus (strain MVP-899) and 14C leucine was added 20 hours after infection for $20 \mathrm{~min}$ (pulse). Then the cells were washed with PBS and the label was chased for 1, 2 and 3 hours. The cells were fractionated as described earlier [1] to get cytosol, membrane and nuclear fractions. Lanes 1 and 6 , protein markers, lanes 2 - 5, cytosol fraction, lanes 7 - 10, membrane fraction. Lanes 2 and 7, pulse labeling, lanes 3 and 8, 4 and 9, 5 and 10, chase for 1, 2 and 3 hours accordingly. The nuclear fraction did not contain viral proteins. (b) 14C leucine was added $40 \mathrm{~h}$ after infection for 30 min. (lanes 1, 3, 6) and the label was chased for $1 \mathrm{~h}$ (lanes 2, $4,7)$ Lanes 5 and 8 , the cells were labeled for $3 \mathrm{~h}$. Lanes 1,2 , cytosol fraction, 3 - 5, membrane fraction, 6 - 8, nuclear fraction.

hybridization [1].

In 293T cells matrix protein has been revealed $40 \mathrm{~h}$ after infection mainly in membrane fraction and in less amount in nuclear fraction (Figure 2(a)). Viral RNA has been also found by PCR in immunoprecipitates of membrane fractions obtained after treatment with antiMA antibody which do not interact with Gag precursor (the primers used for LTR region of HIV-1 genome were L1 5'-g485ggagctctctggctaact-3' and L2 5'-g931gattaactgcgaatcgttc-3') (Figure 2(b)). These data suggest that MA could be involved into viral RNA transmission to plasma membrane.

To confirm the role of MA in viral RNA transmission, we compared the intracellular localization and transport of the complexes containing wild MA with that containing mutant MA. Plasmid containing mutant MA was kindly presented by Dr. S. Dupont who also showed that the virus containing mutant MA is not infectious [2]. The mutant contained two changed amino acids in N-terminal region: lysine in 18 position was changed on alanine and arginine in 22 position on glycine. 293T cells were transfected by the plasmids expressing standart MA or mutant MA, and after 24 hours of incubation the cells were fractionated by the method described by Niederman [6] to

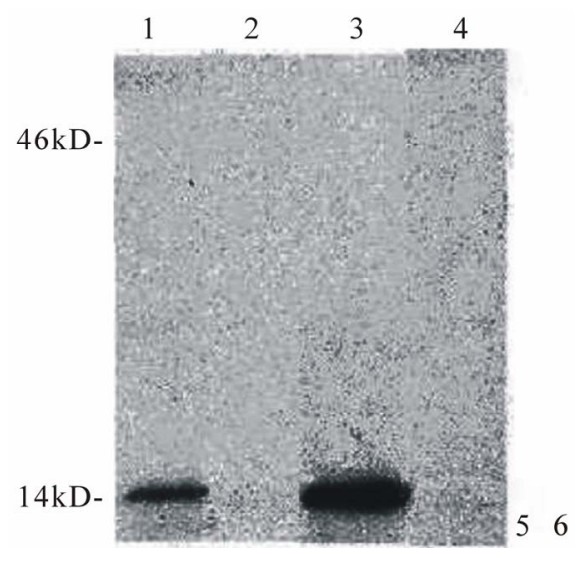

(a)

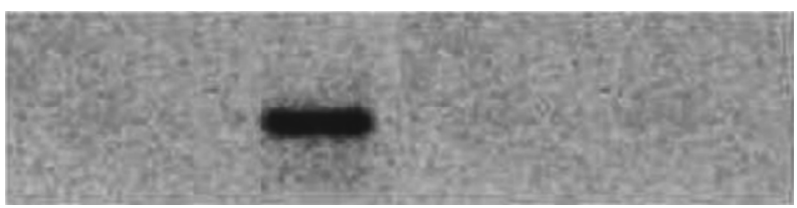

(b)

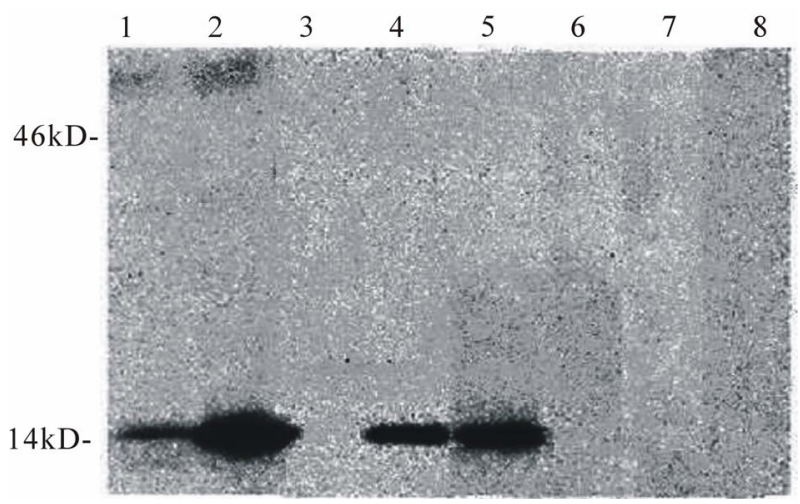

(c)

Figure 2. Intracellular localization of MA and gRNA 24 h after $293 \mathrm{~T}$ cells transfection. Lane 1 , nuclear fraction, lane 2 , cytoskeleton fraction, lane 3 , membrane fraction, lane 4 , cytosol fraction. (c) Localization in cell fractions of MAgRNA complex after transfection of 293T cells with plasmids containing wild MA (lanes 1, 3, 5, 7) and mutant MA (lanes 2, 4, 6, 8). 1, 2, nuclear fraction, 3, 4, cytoskeleton fraction, 5, 6, membrane fraction, 7, 8, cytosol fraction. (a) Immunoblot. (b) PCR using primers LTR-U5-gag to genome RNA. RNA was isolated from immunoprecipitate by MA monoclonal antibody which does not react with Gag precursor. Lanes 1 - 3, PCR using reverse transcription reaction, lanes 4 - 6, control, PCR without preliminary reverse transcription. 
get the fractions of cytosol, membranes, nuclei and cytoskeleton. Localization of MA in cellular fractions was determined using immunoblot.

After transfection with plasmid expressing standard MA, the small amount of MA was found in the nuclear fraction while the main part of the protein was localized in the membrane fraction. However, after transfection with plasmid expressing mutant M4 matrix the protein was not found in the membrane fraction but was localized in the nuclear and cytoskeleton fractions (Figure 2(c)). These data show that the association of the mutant MA with membranes is blocked, probably because mutant MA lost its membranophylic signal (myristic acid) on its $\mathrm{N}$ terminus, and the mutant MA protein associated with viral RNA "sticks" in nuclei and cytoskeleton, the fractions of its previous location. These results allow suggesting that the complex MA-viral RNA is transported from the nuclei to membranes by cytoskeleton. The reverse transport from membranes to nuclei was described for viral reverse transcription and preintegration complexes [7] showing that the virus widely uses cytoskeleton for its intracellular transport.

\section{Conclusions}

Despite the progress in defining determinates of Gag participation in HIV assembly, Gag intracellular interactions and trafficking to the assembly sites in the infected cells are poorly understand. Our results indicate, that MA is connected with viral RNA early after infection and the complex is transported to the cellular membrane-the place of viral assembly. When the mutant MA was used instead of standard MA, the complex of MA and viral RNA was not found in the cellular membrane, but sticks in nucleus and cytoskeleton fractions. These results show that MA-RNA complex is transported from the nuclei to the cellular membrane by cytoskeleton.

The data also show that MA is cleaved from Gag precursor very early after Gag synthesis and thus could be involved into virus assembly as an individual protein.

The significant role of MA in HIV-1 replication shows that this protein is the candidate antigen for therapeutic vaccine against AIDS.

\section{REFERENCES}

[1] A. G. Bukrinskaya, G. K. Vorkunova and Y. Y. Tentsov, "HIV-1 Matrix Protein P17 Resides in Cell Nuclei in Association with Genomic RNA,” AIDS Research and Human Retroviruses, Vol. 8, No. 10, 1992, pp. 1795-1801. doi:10.1089/aid.1992.8.1795

[2] S. Dupont, N. Sharova, C. DeHoratius, C. M. A. Virbasius, X. C. Zhu, A. G. Bukrinskaya, M. Stevenson and M. R. Green, “A Novel Nuclear Export Activity in HIV-1 Matrix Protein Required for Viral Replication,” Nature, Vol. 402, No. 6762, 1999, pp. 681-685. doi:10.1038/45272

[3] A. H. Kaplan and R. Swanstrom, "HIV-1 Gag Proteins Are Processed in Two Cellular Compartments," Proceedings of the National Academy of Sciences of USA, Vol. 88, No. 10, 1991, pp. 4528-4532. doi:10.1073/pnas.88.10.4528

[4] A. Bukrinskaya, "HIV-1 Matrix Protein: A Mysterious Regulator of the Viral Life Cycle,” Virus Research, Vol. 124, No. 1-2, 2007, pp. 1-11. doi:10.1016/j.virusres.2006.07.001

[5] N. Sharova and A. Bukrinskaya, "P17 and P17-Containing Gag Precursor of Input HIV Are Transported into the Nuclei of Infected Cells," AIDS Research and Human Retroviruses, Vol. 7, No. 3, 1991, pp. 303-306. doi:10.1089/aid.1991.7.303

[6] T. M. J. Niederman, W. R. Hastings and L. Ratner, "Myristoylation-Enhanced Binding of the HIV-1 Net Protein to T Cell Skeletal Matrix,” Virology, Vol. 197, No. 1, 1993, pp. 420-425. doi:10.1006/viro.1993.1605

[7] A. Bukrinskaya, B. Brichacek, A. Mann and M. Stevenson, "Establishment of a Functional HIV-1 Reverse Transcription Complex Involves the Cytoskeleton,” The Journal of Experimental Medicine, Vol. 188, No. 11, 1998, pp. 2113-2125. doi:10.1084/jem.188.11.2113 